\title{
Clinical Characteristics of Familial and Sporadic Age- Related Macular Degeneration: Differences and Similarities
}

\author{
Nicole T. M. Saksens, ${ }^{1}$ Eveline Kersten, ${ }^{1}$ Joannes M. M. Groenewoud, ${ }^{2}$ Mark J. J. P. van Grinsven, ${ }^{3}$ \\ Johannes P. H. van de Ven, ${ }^{1}$ Clara I. Sánchez, ${ }^{3}$ Tina Schick, ${ }^{4}$ Sascha Fauser, ${ }^{4}$ Anneke I. den \\ Hollander, ${ }^{1}$ Carel B. Hoyng, ${ }^{1}$ and Camiel J. F. Boon ${ }^{1,5}$
}

${ }^{1}$ Department of Ophthalmology, Radboud University Medical Center, Nijmegen, The Netherlands

${ }^{2}$ Department of Epidemiology, Biostatistics, and Health Technology Assessment, Radboud University Medical Center, Nijmegen, The Netherlands

${ }^{3}$ Diagnostic Image Analysis Group, Radboud University Medical Center, Nijmegen, The Netherlands

${ }^{4}$ Department of Ophthalmology, University Hospital of Cologne, Cologne, Germany

${ }^{5}$ Department of Ophthalmology, Leiden University Medical Center, Leiden, The Netherlands

Correspondence: Camiel J.F. Boon, Department of Ophthalmology, Leiden University Medical Centre, Albinusdreef 2, 2333 ZA Leiden, the Netherlands;

C.J.F.Boon@lumc.n1.

Submitted: April 25, 2014

Accepted: September 29, 2014

Citation: Saksens NTM, Kersten E, Groenewoud JMM, et al. Clinical characteristics of familial and sporadic age-related macular degeneration: differences and similarities. Invest $O p h$ thalmol Vis Sci. 2014;55:7085-7092 DOI:10.1167/iovs.14-14659
Purpose. We describe the differences and similarities in clinical characteristics and phenotype of familial and sporadic patients with age-related macular degeneration (AMD).

Methods. We evaluated data of 1828 AMD patients and 1715 controls enrolled in the European Genetic Database. All subjects underwent ophthalmologic examination, including visual acuity testing and fundus photography. Images were graded and fundus photographs were used for automatic drusen quantification by a machine learning algorithm. Data on disease characteristics, family history, medical history, and lifestyle habits were obtained by a questionnaire.

Results. The age at first symptoms was significantly lower in AMD patients with a positive family history ( 68.5 years) than in those with no family history $\left(71.6\right.$ years, $P=1.9 \times 10^{-5}$ ) Risk factors identified in sporadic and familial subjects were increasing age (odds ratio [OR], 1.08 per year; $P=3.0 \times 10^{-51}$, and OR, $1.15 ; P=5.3 \times 10^{-36}$, respectively) and smoking (OR, 1.01 per pack year; $P=1.1 \times 10^{-6}$ and $\mathrm{OR}, 1.02 ; P=0.005$ ). Physical activity and daily red meat consumption were significantly associated with AMD in sporadic subjects only (OR, $0.49 ; P=3.7 \times 10^{-10}$ and OR, $1.81 ; P=0.001$ ). With regard to the phenotype, geographic atrophy and cuticular drusen were significantly more prevalent in familial AMD (17.5\% and 21.7\%, respectively) compared to sporadic AMD (9.8\% and $12.1 \%)$.

Conclusions. Familial AMD patients become symptomatic at a younger age. The higher prevalence of geographic atrophy and cuticular drusen in the familial AMD cases may be explained by the contribution of additional genetic factors segregating within families.

Keywords: AMD, family history, clinical characteristics

\begin{abstract}
A ge-related macular degeneration (AMD) is a multifactorial retinal disease leading to severe vision loss among the elderly. Advanced age, female sex, smoking, and obesity (body mass index $[\mathrm{BMI}]>30$ ) are most commonly reported as important demographic and environmental risk factors for the development of AMD. ${ }^{1-6}$ In addition, several important genetic variants have been found to be associated with AMD, either as a risk factor or as a protective factor. The strongest associations have been reported for the singlenucleotide polymorphisms (SNPs) in the complement factor $\mathrm{H}$ gene (CFH Y402H; rs1061170), and in the age-related maculopathy susceptibility 2 gene (ARMS2 A69S; rs10490924), which strongly increase the risk of developing AMD. ${ }^{7-11}$

Previous studies have demonstrated aggregation of AMD in families. ${ }^{12,13}$ A family history of AMD has been reported as a significant risk factor for AMD. ${ }^{14}$ Individuals are at a higher risk of developing AMD when a first-degree relative is
\end{abstract}

affected. Moreover, having an affected parent is associated with a higher risk than having an affected sibling. ${ }^{13,14}$ Shahid et al. ${ }^{14}$ showed an odds ratio (OR) for AMD of 27.8 in people with an affected parent and 12.0 for people with an affected sibling. Likewise, Luo et al. ${ }^{13}$ reported a relative risk for the development of AMD of 5.66 for people with an affected parent and 2.95 for people with an affected sibling.

A lower age at onset has been reported in familial AMD patients and heritability of AMD subtypes has been suggested. ${ }^{14,15}$ Even though environmental and genetic risk factors can cluster in families, the number of affected family members in large densely affected families cannot be fully explained by clustering of known risk factors. ${ }^{12}$ Several recent studies have shown that rare, highly penetrant genetic variants can strongly increase the risk of developing AMD in families with AMD, as well as in the AMD population in general. ${ }^{16-20}$ 
Little is known about clinical differences and similarities between patients with and without a family history for AMD. The purpose of this study is to gain more insight into the clinical and phenotypic characteristics of familial and sporadic AMD patients, and to analyze if there are distinct clinical differences between these subgroups.

\section{Patients and Methods}

\section{Study Population}

The European Genetic Database (EUGENDA, available in the public domain at www.eugenda.org) is a multicenter database for clinical and molecular analysis of AMD founded by the Radboud University Medical Center (Nijmegen, the Netherlands) and the Department of Ophthalmology of the University Hospital of Cologne (Cologne, Germany). This database contains data of AMD patients and control individuals, including family history, environmental risk factors, and ophthalmologic examination. For this retrospective study we evaluated data of 1828 Caucasian patients with AMD and 1715 Caucasian controls enrolled in EUGENDA of whom family history of AMD, smoking status, BMI, age, and sex data were available.

This study was performed in accordance with the tenets of the Declaration of Helsinki and was approved by the local ethical committees at the Radboud University Medical Center and the University of Cologne. Written informed consent was obtained from all participants before enrolling in EUGENDA.

\section{Questionnaire, Clinical Evaluation, and Grading}

Before enrollment in the EUGENDA database, all subjects were interviewed with a detailed questionnaire about disease characteristics (e.g., age at first symptoms), family history, medical history, and lifestyle habits, such as smoking status, diet, and physical activity. For each subject, BMI was calculated using body height and body weight as reported in the questionnaire. Based on years of smoking and number of cigarettes smoked per day, we calculated the number of pack years. Each subject underwent an ophthalmologic examination, including Early Treatment Diabetic Retinopathy Study (ETDRS) visual acuity testing, dilated fundus examination, and color fundus photography. The best corrected visual acuity (BCVA) was converted to logMAR visual acuity for the purpose of statistical analysis. Two independent certified reading center graders evaluated color fundus photographs of both eyes of all subjects according to the standard protocol of the Cologne Image Reading Center and Laboratory (CIRCL). ${ }^{21}$ Digital nonstereoscopic $30^{\circ}$ color fundus photographs centered on the fovea were performed with a Topcon TRC 50IX camera (Topcon Corporation, Tokyo, Japan). The diagnosis and grading of AMD was based on a classification and grading scheme as described previously. $^{22}$ For all analyses in this study we used the grading of the worst affected eye, and subjects with only one gradable color fundus photograph were excluded. Additionally, in 1184 AMD subjects spectral-domain optical coherence tomography (SD-OCT, Spectralis; Heidelberg Engineering, Heidelberg, Germany) was available and evaluated for the presence of reticular pseudodrusen. In 677 subjects the presence of cuticular drusen was evaluated based on available fluorescein angiography, performed using the Spectralis HRA system (Heidelberg Engineering). ${ }^{23}$ The SD-OCT volume scans consisting of 19 or 37 parallel OCT Bscans were used for analysis, covering $6 \times 4 \mathrm{~mm}$ of the macula. For each OCT B-scan, 20 images were averaged using the automated real-time function. ${ }^{21}$ Evaluation of the presence of reticular pseudodrusen on SD-OCT and cuticular drusen on fluorescein angiography was done by one senior grader.

Based on diagnosis and family history, the participants in this study were divided into four groups: sporadic AMD, sporadic control, familial AMD, and familial control. We classified subjects as familial in case of confirmed or possible AMD in at least one close relative, defined as a parent, sibling, or child. Sporadic subjects were defined as individuals who did not have a close relative with AMD.

\section{Automatic Drusen Quantification}

In addition to the human grading of AMD based on photographs, a machine learning algorithm for computeraided diagnosis of AMD was used for detection and quantification of drusen number and area (measured in pixels). This was described previously as accurate in detecting and quantifying drusen number and area on color fundus photographs of patients with nonadvanced AMD and control subjects. ${ }^{24}$ Patients with advanced AMD in the worst affected eye have been excluded for this specific analysis, because the automatic system was not designed to deal with images containing signs of advanced stage AMD. A quality score ranging from 0 to 1 was calculated, with 0 being the worst quality and 1 being the best quality. Only color fundus photographs were selected with a quality score of 0.3 or more, which corresponds to sufficient quality for human grading.

\section{Statistical Analysis}

All statistical analyses were performed using IBM SPSS Statistics software, version 20.0 (Released 2011; IBM Corp., Armonk, NY, USA). Each potential risk factor for the development of AMD obtained from the questionnaire was included separately in a logistic regression analysis adjusted for age, sex, smoking, and BMI. The ORs were calculated for familial subjects (familial AMD versus familial controls) and sporadic subjects (sporadic AMD versus sporadic controls). Significant differences between ORs for sporadic and familial subjects were identified by interaction analysis using binary logistic regressions. All continuous variables were analyzed using an independent sample $t$-test or 1-way ANOVA. An univariate general linear model was used when continuous variables were analyzed with correction for other variables. Categorical variables were analyzed using a $\chi^{2}$ test. Differences with a $P$ value less than 0.05 were considered statistically significant. Because multiple possible risk factors were analyzed and many tests of significance were performed in our study, Bonferroni correction was performed for the risk and interaction analysis of environmental factors.

\section{Results}

Demographic characteristics of the cohort are shown in Table 1. All four groups were comparable for sex, smoking, and BMI. The mean age of the familial subjects was slightly lower than in sporadic subjects (69.6 and 73.0 years, respectively; $P=4.7 \times$ $10^{-16}$ ), mainly due to younger familial control individuals. In 309 subjects who reported in the questionnaire to have a close relative with (possible) AMD, the ophthalmologically examined AMD status of close relatives was available. To determine the degree of misclassification of subjects into familial or sporadic based on the questionnaire, we compared the family history to 
TABLE 1. Demographic Characteristics

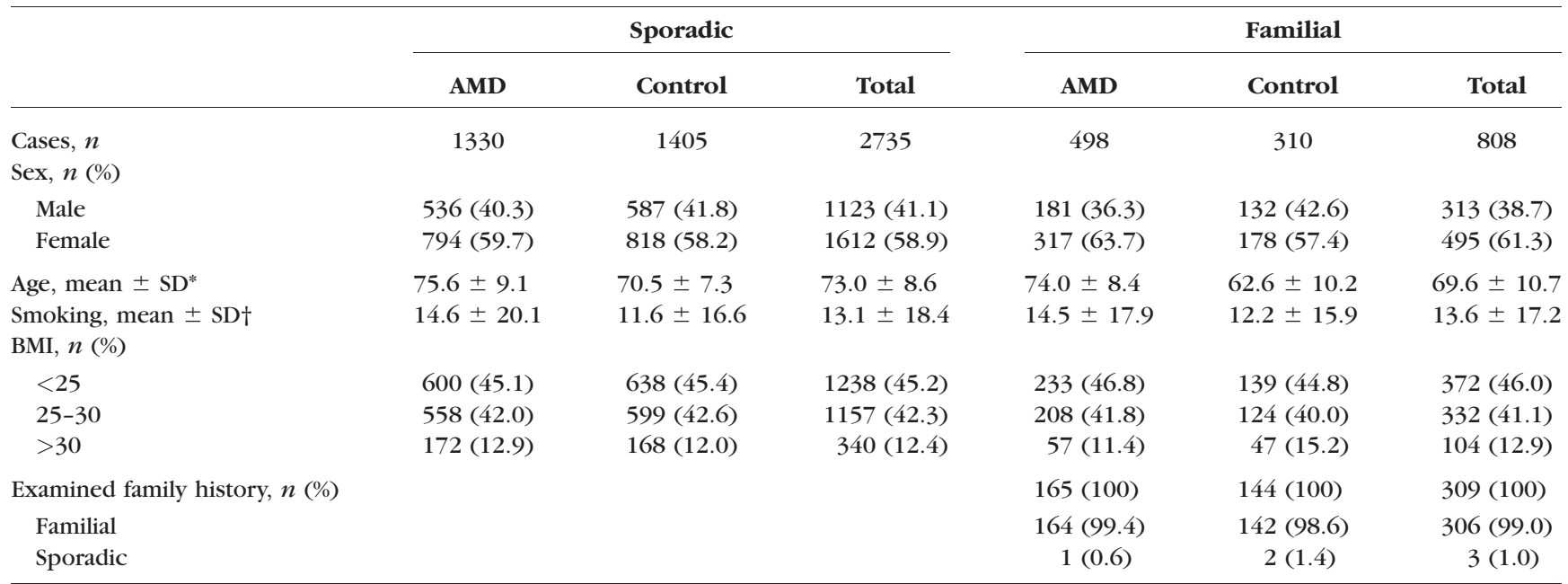

The study included 498 familial AMD patients deriving from 393 families (393 probands and 105 family members) and 310 controls deriving from 216 families (216 probands and 94 family members). In 309 familial cases information about ophthalmologic examination in their close relatives (parents, sibling, and/or children) was available and was compared to family history based on the questionnaire to determine the degree of misclassification. Familial, positive family history for AMD (confirmed or possible AMD in at least one close relative [parent, sibling or child]); sporadic, no positive family history.

$*$ Age of participation in years.

† Smoking in pack years.

these examined data. In 3 of 309 cases where subjects reported in the questionnaire to have at least one close relative with AMD, no family members seemed to be affected upon ophthalmological examination. Therefore, these cases were incorrectly classified as familial. No ophthalmological information was available for relatives of sporadic subjects included in this study.

\section{Familial and Sporadic AMD: Clinical Differences and Similarities}

Information about the age at first symptoms was available in 703 AMD patients (469 sporadic subjects, 234 familial subjects, Table 2$)$. The age at first symptoms was significantly lower $\left(P=1.9 \times 10^{-5}\right)$ in familial AMD patients (mean, 68.5 years; SD, 9.8) than in sporadic AMD patients (mean, 71.6 years; SD, 8.7) with a mean difference of 3.1 years $(95 \%$ confidence interval [CI], 1.7-4.5). When subdividing AMD in the presence or absence of the reticular pseudodrusen subtype, the age at first symptoms was also lower in familial AMD patients. In addition, AMD patients with reticular pseudodrusen have a significantly higher age at first symptoms than patients without reticular pseudodrusen, in sporadic and familial patients $\left(P=4.4 \times 10^{-10}\right.$ and $P=$ 0.002 , respectively). In contrast, in AMD patients with the cuticular drusen subtype, the age at first symptoms was lower than in patients without cuticular drusen, in sporadic and familial patients $\left(P=1.26 \times 10^{-6}\right.$ and $P=0.074$, respectively). However, no significant difference in age at first symptoms was observed between familial and sporadic patients with the cuticular drusen subtype $(P=0.740)$.

Despite a younger age at first symptoms, BCVA of both eyes did not differ significantly between familial and sporadic AMD patients when subdivided in three age categories (data shown in Table 2). Also, if young patients ( $<60$ years) were analyzed separately, no difference in BCVA was observed between familial and sporadic patients (data not shown). After distinguishing between advanced and nonadvanced AMD subjects, BCVA also was comparable between sporadic and familial patients.

To identify risk factors in our cohort, we analyzed several demographic and environmental factors (Table 3). Risk factors identified in sporadic and familial AMD patients were increasing age $\left(P=3.0 \times 10^{-51}\right.$ and $\left.P=5.3 \times 10^{-36}\right)$ and smoking $\left(P=1.1 \times 10^{-6}\right.$ and $\left.P=0.005\right)$. Interaction analysis showed a significant difference between sporadic and familial subjects for increasing age $\left(P=9.4 \times 10^{-7}\right)$.

In terms of comorbidity (Table 3 ), allergy was significantly associated with a decreased risk of AMD for sporadic and familial subjects $(P=0.002$ and $P=0.024$, respectively). Diabetes mellitus was a risk factor for the development of AMD in sporadic patients (OR, 1.34; 95\% CI, 1.02-1.75; $P=$ $0.035)$, but not in familial cases $(P=0.704)$. No significant interaction between family history and allergy or diabetes was present. Cardiovascular disease, renal disease, and autoimmune disease were no significant risk factors for AMD in our cohort.

With regard to dietary factors, we observed that eating red meat a few times per week or daily is a significant risk factor in sporadic AMD patients (OR, 1.24; 95\% CI, 1.05-1.48; $P=0.013$ and $\mathrm{OR}, 1.81 ; 95 \% \mathrm{CI}, 1.30-2.54 ; P=0.001$, respectively), but not in familial subjects. A protective factor for AMD in sporadic patients was eating fruit a few times per week (OR, 0.60; 95\% CI, $0.40-0.90 ; P=0.013)$. Intake of fruit every day did not seem to further decrease the risk of AMD (OR, 0.74; 95\% CI, 0.521.04; $P=0.085)$. However, consumption of fruit was not significantly associated with a decreased risk of AMD in familial subjects.

Regular physical activity, one or two times a week, was significantly associated with a decreased risk for AMD in the sporadic AMD subgroup (OR, 0.67; 95\% CI, 0.56-0.81; $P=2.6$ $\times 10^{-6}$ ) and the familial AMD subgroup (OR, 0.62; $95 \% \mathrm{CI}$, 0.42-0.94; $P=0.022$ ).

After Bonferroni correction of the demographic and environmental risk factors for AMD, the association of increasing age with AMD in familial and sporadic subjects remained significant, as well as the association of female sex, 
TABLE 2. Clinical Features and Staging of Sporadic and Familial AMD Patients

\begin{tabular}{|c|c|c|c|}
\hline & $\begin{array}{c}\text { Sporadic AMD } \\
\text { Mean } \pm \text { SD }\end{array}$ & $\begin{array}{c}\text { Familial AMD } \\
\text { Mean } \pm \text { SD }\end{array}$ & $\boldsymbol{P}$ \\
\hline \multicolumn{4}{|l|}{ Age at first symptoms, y } \\
\hline AMD, total* & $71.6 \pm 8.7$ & $68.5 \pm 9.8$ & $1.9 \times 10^{-5}$ \\
\hline AMD, reticular pseudodrusen $\dagger$ & $76.2 \pm 7.2$ & $72.5 \pm 7.3$ & 0.008 \\
\hline AMD, cuticular drusen $\neq$ & $65.8 \pm 9.2$ & $64.5 \pm 17.7$ & 0.740 \\
\hline \multicolumn{4}{|l|}{ Visual acuity per age category $₫$} \\
\hline$<70$ & $0.12 \pm 0.27$ & $0.09 \pm 0.25$ & 0.293 \\
\hline $70-80$ & $0.19 \pm 0.29$ & $0.26 \pm 0.36$ & 0.107 \\
\hline$>80$ & $0.40 \pm 0.40$ & $0.38 \pm 0.46$ & 0.814 \\
\hline \multicolumn{4}{|l|}{ Visual acuity per stage $₫$} \\
\hline Nonadvanced & $0.05 \pm 0.14$ & $0.03 \pm 0.13$ & 0.367 \\
\hline Advanced & $0.33 \pm 0.37$ & $0.37 \pm 0.42$ & 0.314 \\
\hline \multicolumn{4}{|l|}{ Grading, $n(\%)$} \\
\hline Early & $301(22.6)$ & $94(18.9)$ & 0.158 \\
\hline Intermediate & $250(18.8)$ & $90(18.1)$ & \\
\hline Advanced & 779 (58.6) & $314(63.1)$ & \\
\hline GA & $76(9.8)$ & $55(17.5)$ & $3.0 \times 10^{-4}$ \\
\hline $\mathrm{CNV}$ & $660(84.7)$ & $234(74.5)$ & \\
\hline Mixed & $43(5.5)$ & $25(8.0)$ & \\
\hline
\end{tabular}

* Data on age of first symptoms were available in 469 sporadic and 234 familial AMD patients.

† Data on age of first symptoms were available in 75 sporadic and 45 familial AMD patients with reticular pseudodrusen.

‡ Data on age of first symptoms were available in 32 sporadic and 19 familial AMD patients with cuticular drusen.

$\S$ Visual acuity defined as best-corrected logMAR visual acuity.

smoking, allergy, daily red meat consumption and physical activity in sporadic patients only.

\section{Familial and Sporadic AMD: Phenotypic Differences and Similarities}

The prevalence of early, intermediate, and advanced stage AMD was similar in the familial and sporadic AMD patient group (Table 2). After differentiation of advanced AMD into geographic atrophy (GA), choroidal neovascularization (CNV), and mixed (GA and $\mathrm{CNV}$ in one patient), we found that GA was more prevalent in familial AMD patients $(17.5 \%)$ than in sporadic AMD patients $\left(9.8 \%, P=3.0 \times 10^{-4}\right)$, despite a comparable SNP load of $C F H$ Y402H and ARMS2 A69S between familial $(n=51)$ and sporadic patients $(n=58)$ with GA (data not shown). In 829 sporadic subjects and 355 familial subjects data on reticular pseudodrusen were available, and data on cuticular drusen were available in 520 sporadic and 157 familial subjects. The prevalence of reticular pseudodrusen was comparable between familial (18.0\%) and sporadic subjects $(18.8 \%, P=0.749)$, whereas the prevalence of cuticular drusen was significantly higher in familial AMD (21.7\%) compared to sporadic AMD (12.1\%, $P=$ $0.003)$.

Data on the number of drusen and area of drusen within the macular area were available for 689 sporadic subjects and 203 familial subjects (Table 4). Familial subjects showed a trend toward a higher number of drusen and a larger area of drusen in the macula compared to sporadic patients, although this was only significant for the area of drusen in subjects with intermediate AMD $(P=0.043)$. After correction for age, sex, $\mathrm{BMI}$, and smoking, the mean area of drusen in sporadic subjects with intermediate AMD was 1114.49 and 1415.63 in familial subjects, which were no longer significantly different $(P=0.160)$.

\section{Discussion}

\section{Familial and Sporadic AMD: Clinical Differences and Similarities}

Familial AMD patients have a lower age at first symptoms compared to sporadic AMD patients. The phenomenon of a lower age at onset in patients with familial occurrence has been shown in other complex diseases with a significant genetic component, such as schizophrenia and Alzheimer's disease. ${ }^{25,26}$ A lower age at onset in familial AMD patients has previously been reported by Shahid et al. ${ }^{14}$ (70.4 years in familial patients and 73.2 years in sporadic patients), and is in accordance with the mean difference of 3.1 years in our study. A significant difference in age of onset between familial and sporadic subjects also was observed in AMD patients with reticular pseudodrusen, but not in patients with cuticular drusen. However, as a result of a positive family history, familial subjects may have an increased awareness of visual symptoms which can lead to an earlier visit at a physician for evaluation. Therefore, it should be noted that the lower age at first symptoms in familial AMD patients may be partially attributed to an ascertainment bias.

In our study, BCVA per age category did not differ between familial and sporadic AMD patients, suggesting that visual acuity does not decrease earlier or at a faster rate in familial patients, despite the lower age at first symptoms in familial AMD patients. Heightened awareness in familial patients may explain why no actual difference in BCVA was observed.

Similar to other studies, ${ }^{1-3,6}$ smoking and advanced age were associated with the development of AMD in the current study, in sporadic and familial subjects. Furthermore, age was a more important risk factor for AMD in familial subjects as age shows a significant interaction with family history, resulting in a younger age at onset in familial subjects. 





TABLE 4. Number and Area of Drusen in Sporadic and Familial Control Individuals and Nonadvanced AMD Patients

\begin{tabular}{|c|c|c|c|c|c|}
\hline & \multicolumn{2}{|r|}{ Sporadic } & \multicolumn{2}{|r|}{ Familial } & \multirow[b]{2}{*}{$\boldsymbol{P}$} \\
\hline & $\boldsymbol{n}$ & Mean \pm SD & $n$ & Mean \pm SD & \\
\hline \multicolumn{6}{|l|}{ Control } \\
\hline Drusen, $n$ & 159 & $2.25 \pm 6.0$ & 24 & $2.85 \pm 3.4$ & 0.633 \\
\hline Drusen area* & 159 & $81.65 \pm 149.6$ & 24 & $128.86 \pm 129.5$ & 0.145 \\
\hline \multicolumn{6}{|l|}{ Early } \\
\hline Drusen, $n$ & 291 & $8.69 \pm 20.0$ & 93 & $11.69 \pm 27.37$ & 0.254 \\
\hline Drusen area* & 291 & $219.17 \pm 353.4$ & 93 & $249.67 \pm 416.6$ & 0.489 \\
\hline \multicolumn{6}{|l|}{ Intermediate } \\
\hline Drusen, $n$ & 239 & $40.25 \pm 62.4$ & 86 & $46.37 \pm 48.9$ & 0.411 \\
\hline Drusen area* & 239 & $1167.90 \pm 1735.4$ & 86 & $1598.70 \pm 1555.4$ & 0.043 \\
\hline
\end{tabular}

* Area of drusen in pixels.

Ristau et al. ${ }^{22}$ have reported recently that allergy is associated with a reduced risk of AMD. We did not find a significant difference for the association of allergy with AMD between familial and sporadic subjects, so the protective effect of allergy does not seem to be influenced by family history.

The pathogenesis of AMD as well as cardiovascular disease and diabetes have been linked to oxidative stress, inflammation and a vascular origin. Moreover, diabetes, cardiovascular disease, and its risk factors have been associated with the development of AMD, although this was not consistent among studies. ${ }^{1,27-30}$ In the current study we observed that sporadic subjects with diabetes have an increased risk for AMD. However, diabetes was no risk factor for familial AMD, which may be explained by the larger genetic component in the pathophysiology of familial AMD, while sporadic AMD may be associated with a larger contribution of environmental or lifestyle factors, such as diabetes (and associated factors).

Several studies reported an increased risk of AMD for patients with chronic renal disease. ${ }^{31,32}$ It has been shown that AMD and renal diseases, such as membranoproliferative glomerulonephritis type 2 and atypical hemolytic uremic syndrome, are related to the same genetic variants of the complement pathway, including the complement factor $\mathrm{H}$ gene. 33-35 Therefore, we evaluated whether renal disease might be correlated with AMD in familial subjects. However, we did not find a clear association between familial AMD and renal disease in our study population, possibly due to the low number of patients with renal disease.

We also compared dietary factors between familial and sporadic subjects. It is interesting to investigate these dietary factors, since these are modifiable. The consumption of red meat at least a few times a week increased the risk of AMD in our sporadic patient cohort. This is supported by findings of Chong et al., ${ }^{36}$ demonstrating that higher red meat intake was associated with the development of AMD. In our study consumption of fruit a few times a week was associated with a decreased risk for the development of AMD, but we did not observe such an association with frequent consumption of vegetables. In agreement with this finding, Cho et al. ${ }^{37}$ and Zerbib et al. ${ }^{38}$ described a protective effect of frequent consumption of fruits for exudative AMD, but no association with the consumption of vegetables. A study by Seddon et al. ${ }^{39}$ showed that intake of foods rich in carotenoids, in particular green leafy vegetables, decreased the risk of exudative AMD. Our study might be limited because we did not discriminate between different kinds of vegetables and no risk calculation was performed for the progression to advanced AMD. This may explain why we did not observe a protective effect for the consumption of vegetables. Also, it must be considered that fruit consumption can be related to a more healthy lifestyle in general, and, therefore, these results may be confounded by other factors, other than smoking and BMI, related to a healthy lifestyle.

In cuticular drusen, a clinical subtype of AMD that tends to cluster in families, differences in environmental and genetic risk factors have been reported compared to the AMD group as a whole. ${ }^{23,40}$ We previously reported that the association with smoking was significantly lower in patients with cuticular drusen compared to AMD patients without cuticular drusen. ${ }^{23}$ In this study, we observed no significant difference in environmental risk factors, such as smoking, between familial and sporadic AMD. However, several factors, such as the consumption of red meat and frequent physical activity, tended to have a less important role in familial AMD than in sporadic AMD, supporting a stronger genetic component in the pathophysiology of AMD in families.

\section{Familial and Sporadic AMD: Phenotypic Differences and Similarities}

In our study population, GA was more prevalent in familial AMD patients than in sporadic patients. This cannot be explained by selection bias of familial patients, because if only the probands of the familial group $(n=262)$ were included in the analysis, GA was still significantly more prevalent in the familial AMD group than in the sporadic group (17.6\% and $9.8 \%$, respectively, $P=0.001$ ). This finding may be explained by the stronger influence of genetic factors in the pathogenesis of familial AMD in certain phenotypic subtypes, such as GA. Previously Shahid et al. ${ }^{14}$ suggested heritability of AMD subtypes, but these investigators were not able to confirm this hypothesis due to low numbers of subjects. Sobrin et al. ${ }^{15}$ showed that siblings are more likely to develop the same advanced subtype as their proband. This may suggest the contribution of genetic variants in these familial patients, ${ }^{16,35,41}$ which may increase the risk for developing GA rather than CNV. Affected members of densely affected families have been reported to bear a lower SNP load than expected based on five common known AMD risk variants; $C F H$ (rs1061170 and rs1410996), ARMS2 (rs10490924), C2-CFB (rs641153 and rs9332739). ${ }^{10}$ This supports the hypothesis that rare genetic variants in these families may explain the high prevalence of AMD.

In this study, we reported a higher prevalence of cuticular drusen in familial AMD compared to sporadic AMD, which is in agreement with previous reports. ${ }^{40,42,43}$ However, in our cohort the prevalence of cuticular drusen was higher and $32 \%$ of the patients with cuticular drusen had a positive family history of AMD compared to $44 \%$ of the patients in a study of Grassi et al. ${ }^{40}$ Previously, our group demonstrated that heterozygous loss-of-function mutations in the $\mathrm{CFH}$ gene are found among family members with cuticular drusen. ${ }^{35,41}$ In addition, rare highly penetrant variants in the $C F I$ gene have been identified in patients with cuticular drusen. ${ }^{16,44}$ Therefore, the higher prevalence of cuticular drusen in families may be explained by segregation of rare, highly penetrant variants within these families.

In addition to a possible ascertainment bias, caused by an increased awareness of disease-associated visual symptoms in familial subjects, another limitation of this study is the classification of subjects into familial or sporadic based on the family history in the questionnaire, which may lead to misclassification. However, in a subset of the familial cases 
ophthalmologic examination data of their close relatives were available and compared to our classification based on the family history of the questionnaire. The degree of misclassification of the family history was very low in the familial subjects, as in only three cases $(1.0 \%)$ no close relative with AMD was found by ophthalmologic examination. Unfortunately, no clinical data of family members of sporadic cases were available. The rate of misclassification may be higher in sporadic individuals, as these subjects may not have been informed of the eye disease of close relatives or the relatives were asymptomatic and therefore, not yet diagnosed with AMD.

In conclusion, this study demonstrated that familial AMD patients have a lower age at first symptoms compared to sporadic patients. Our findings also indicate that familial AMD patients differ from sporadic patients in terms of risk factors and clinical features. The higher prevalence of GA and cuticular drusen in familial AMD patients may be explained by the contribution of additional genetic factors segregating within these families.

\section{Acknowledgments}

Supported by Foundation Fighting Blindness USA (Grant C-GE0811-0548-RAD04), Salentein fellowship, MD Fonds, Oogfonds, Algemene Nederlandse Vereniging ter Voorkoming van Blindheid, Stichting Nederlands Oogheelkundig Onderzoek, and Gelderse Blindenstichting. The authors alone are responsible for the content and writing of the paper.

Disclosure: N.T.M. Saksens, None; E. Kersten, None; J.M.M. Groenewoud, None; M.J.J.P. van Grinsven, None; J.P.H. van de Ven, None; C.I. Sánchez, None; T. Schick, None; S. Fauser, None; A.I. den Hollander, None; C.B. Hoyng, None; C.J.F. Boon, None

\section{References}

1. Chakravarthy U, Wong TY, Fletcher A, et al. Clinical risk factors for age-related macular degeneration: a systematic review and meta-analysis. BMC Ophthalmol. 2010;10:31.

2. Age-Related Eye Disease Study Research G. Risk factors associated with age-related macular degeneration. A casecontrol study in the age-related eye disease study: Age-Related Eye Disease Study Report Number 3. Ophthalmology 2000; 107:2224-2232.

3. Evans JR. Risk factors for age-related macular degeneration. Prog Retin Eye Res. 2001;20:227-253.

4. Clemons TE, Milton RC, Klein R, Seddon JM, Ferris FL III. AgeRelated Eye Disease Study Research G. Risk factors for the incidence of advanced age-related macular degeneration in the Age-Related Eye Disease Study (AREDS) AREDS report no. 19. Opbthalmology. 2005;112:533-539.

5. Seddon JM, Cote J, Davis N, Rosner B. Progression of agerelated macular degeneration: association with body mass index, waist circumference, and waist-hip ratio. Arch Ophthalmol. 2003;121:785-792.

6. Erke MG, Bertelsen G, Peto T, Sjolie AK, Lindekleiv H, Njolstad I. Cardiovascular risk factors associated with age-related macular degeneration: the Tromso Study. Acta Opbthalmologica. 2014;92:662-669.

7. Edwards AO, Ritter R III, Abel KJ, Manning A, Panhuysen C, Farrer LA. Complement factor $\mathrm{H}$ polymorphism and agerelated macular degeneration. Science. 2005;308:421-424.

8. Klein RJ, Zeiss C, Chew EY, et al. Complement factor $\mathrm{H}$ polymorphism in age-related macular degeneration. Science. 2005;308:385-389.

9. Rivera A, Fisher SA, Fritsche LG, et al. Hypothetical LOC387715 is a second major susceptibility gene for age- related macular degeneration, contributing independently of complement factor H to disease risk. Hum Mol Genet. 2005; 14:3227-3236.

10. Sobrin L, Maller JB, Neale BM, et al. Genetic profile for five common variants associated with age-related macular degeneration in densely affected families: a novel analytic approach. Eur J Hum Genet. 2010;18:496-501.

11. Shastry BS. Assessment of the contribution of the LOC 387715 gene polymorphism in a family with exudative age-related macular degeneration and heterozygous $\mathrm{CFH}$ variant $(\mathrm{Y} 402 \mathrm{H})$. J Hum Genet. 2007;52:384-387.

12. Klaver CC, Wolfs RC, Assink JJ, van Duijn CM, Hofman A, de Jong PT. Genetic risk of age-related maculopathy. Populationbased familial aggregation study. Arch Ophthalmol. 1998;116: 1646-1651.

13. Luo L, Harmon J, Yang X, et al. Familial aggregation of agerelated macular degeneration in the Utah population. Vision Res. 2008;48:494-500.

14. Shahid H, Khan JC, Cipriani V, et al. Age-related macular degeneration: the importance of family history as a risk factor. Br J Ophthalmol. 2012;96:427-431.

15. Sobrin L, Ripke S, Yu Y, et al. Heritability and genome-wide association study to assess genetic differences between advanced age-related macular degeneration subtypes. Ophthalmology. 2012;119:1874-1885.

16. van de Ven JP, Nilsson SC, Tan PL, et al. A functional variant in the CFI gene confers a high risk of age-related macular degeneration. Nat Genet. 2013;45:813-817.

17. Helgason H, Sulem P, Duvvari MR, et al. A rare nonsynonymous sequence variant in $\mathrm{C} 3$ is associated with high risk of agerelated macular degeneration. Nat Genet. 2013;45:1371-1374.

18. Raychaudhuri S, Iartchouk $\mathrm{O}$, Chin $\mathrm{K}$, et al. A rare penetrant mutation in $\mathrm{CFH}$ confers high risk of age-related macular degeneration. Nat Genet. 2011;43:1232-1236.

19. Seddon JM, Yu Y, Miller EC, et al. Rare variants in CFI, C3 and C9 are associated with high risk of advanced age-related macular degeneration. Nat Genet. 2013;45:1366-1370.

20. Zhan $\mathrm{X}$, Larson DE, Wang $\mathrm{C}$, et al. Identification of a rare coding variant in complement 3 associated with age-related macular degeneration. Nat Genet. 2013;45:1375-1379.

21. Mokwa NF, Ristau T, Keane PA, Kirchhof B, Sadda SR, Liakopoulos S. Grading of age-related macular degeneration: comparison between color fundus photography, fluorescein angiography, and spectral domain optical coherence tomography. J Ophthalmol. 2013;2013:385915.

22. Ristau T, Ersoy L, Lechanteur Y, et al. Allergy is a protective factor against age-related macular degeneration. Invest Ophthalmol Vis Sci. 2014;55:210-214.

23. van de Ven JP, Smailhodzic D, Boon CJ, et al. Association analysis of genetic and environmental risk factors in the cuticular drusen subtype of age-related macular degeneration. Mol Vis. 2012;18:2271-2278.

24. van Grinsven MJ, Lechanteur YT, van de Ven JP, et al. Automatic drusen quantification and risk assessment of agerelated macular degeneration on color fundus images. Invest Ophthalmol Vis Sci. 2013;54:3019-3027.

25. Suvisaari JM, Haukka J, Tanskanen A, Lonnqvist JK. Age at onset and outcome in schizophrenia are related to the degree of familial loading. Br J Psychiatry. 1998;173:494-500.

26. Duara R, Lopez-Alberola RF, Barker WW, et al. A comparison of familial and sporadic Alzheimer's disease. Neurology. 1993;43: 1377-1384.

27. Tan JS, Mitchell P, Smith W, Wang JJ. Cardiovascular risk factors and the long-term incidence of age-related macular degeneration: the Blue Mountains Eye Study. Ophthalmology. 2007; 114:1143-1150. 
28. Klein R, Klein BE, Tomany SC, Cruickshanks KJ. The association of cardiovascular disease with the long-term incidence of age-related maculopathy: the Beaver Dam eye study. Ophthalmology. 2003;110:636-643.

29. Mitchell P, Wang JJ. Diabetes, fasting blood glucose and agerelated maculopathy: the Blue Mountains Eye Study. Aust N ZJ Ophthalmol. 1999;27:197-199.

30. Klein R, Klein BE, Moss SE. Diabetes, hyperglycemia, and agerelated maculopathy. The Beaver Dam Eye Study. Ophthalmology. 1992;99:1527-1534.

31. Liew G, Mitchell P, Wong TY, Iyengar SK, Wang JJ. CKD increases the risk of age-related macular degeneration. $J \mathrm{Am}$ Soc Nephrol. 2008;19:806-811.

32. Klein R, Knudtson MD, Lee KE, Klein BE. Serum cystatin C level, kidney disease markers, and incidence of age-related macular degeneration: the Beaver Dam Eye Study. Arch Ophthalmol. 2009;127:193-199.

33. Nitsch D, Douglas I, Smeeth L, Fletcher A. Age-related macular degeneration and complement activation-related diseases: a population-based case-control study. Ophthalmology. 2008; 115:1904-1910.

34. Boon CJ, van de Kar NC, Klevering BJ, et al. The spectrum of phenotypes caused by variants in the CFH gene. Mol Immunol. 2009;46:1573-1594.

35. van de Ven JP, Boon CJ, Fauser S, et al. Clinical evaluation of 3 families with basal laminar drusen caused by novel mutations in the complement factor H gene. Arch Ophthalmol. 2012; 130:1038-1047.
36. Chong EW, Simpson JA, Robman LD, et al. Red meat and chicken consumption and its association with age-related macular degeneration. Am J Epidemiol. 2009;169:867-876.

37. Cho E, Seddon JM, Rosner B, Willett WC, Hankinson SE. Prospective study of intake of fruits, vegetables, vitamins, and carotenoids and risk of age-related maculopathy. Arch Ophthalmol. 2004;122:883-892.

38. Zerbib J, Delcourt C, Puche N, et al. Risk factors for exudative age-related macular degeneration in a large French casecontrol study. Graefes Arch Clin Exp Ophthalmol. 2013;252: 899-907.

39. Seddon JM, Ajani UA, Sperduto RD, et al. Dietary carotenoids, vitamins $\mathrm{A}, \mathrm{C}$, and $\mathrm{E}$, and advanced age-related macular degeneration. Eye Disease Case-Control Study Group. JAMA. 1994;272:1413-1420.

40. Grassi MA, Folk JC, Scheetz TE, Taylor CM, Sheffield VC, Stone EM. Complement factor $\mathrm{H}$ polymorphism p.Tyr $402 \mathrm{His}$ and cuticular drusen. Arch Ophthal. 2007;125:93-97.

41. Boon CJ, Klevering BJ, Hoyng CB, et al. Basal laminar drusen caused by compound heterozygous variants in the $\mathrm{CFH}$ gene. Am J Hum Genet. 2008;82:516-523.

42. Lerche W. [Pigment epithelium changes in drusen of the macular region]. Ber Zusammenkunft Dtsch Ophthalmol Ges. 1975;439-446.

43. Barbazetto IA, Yannuzzi NA, Klais CM, et al. Pseudo-vitelliform macular detachment and cuticular drusen: exclusion of 6 candidate genes. Ophthalmic Genet. 2007;28:192-197.

44. Boon CJ, van de Ven JP, Hoyng CB, den Hollander AI, Klevering BJ. Cuticular drusen: stars in the sky. Prog Retin Eye Res. 2013; 37:90-113. 